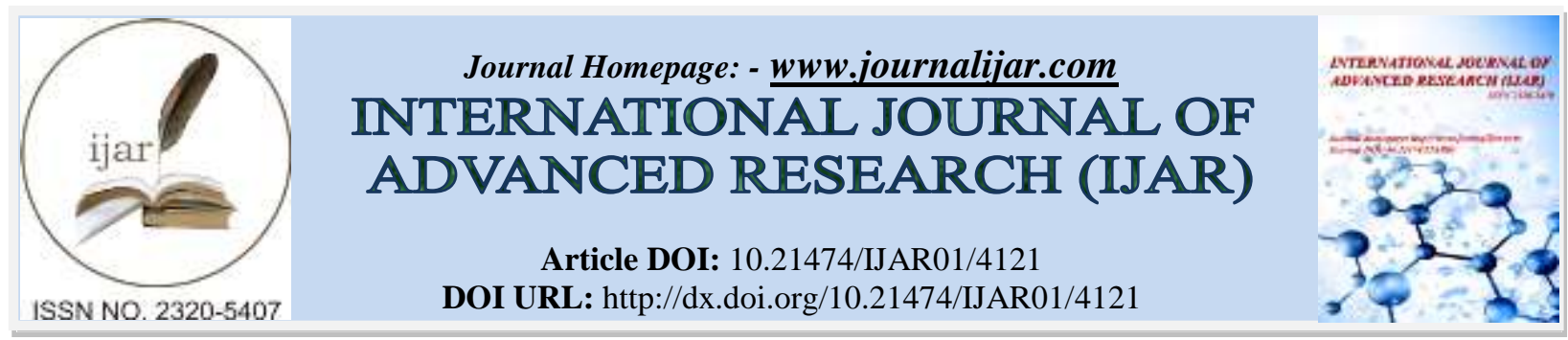

RESEARCH ARTICLE

\title{
ORAL SOMATOSENSORY PERCEPTION IN SKELETAL ANTERIOR OPEN BITE MALOCCLUSION
}

\author{
Prasad Sabarinath ${ }^{1,2^{*}}$, Ravindran Sreeja ${ }^{3}$, Wali Othman ${ }^{4}$, Bajrai Samar ${ }^{5}$ and Ponnan Soumya ${ }^{6}$. \\ 1. PhD student, Department of Oral Sciences, University of Otago, Dunedin, NZ \\ 2. Former faculty, Department of Orthodontics, Ibn Sina National College \\ 3. Assistant Professor, Department of Oral Pathology, Ibn Sina National College \\ 4. Vice Dean, Dental Program, Ibn Sina National College \\ 5. Staff In-Charge, Dental Interns, Ibn Sina National College \\ 6. Assistant Professor, Department of Biostatistics, Ibn Sina National College
}

\section{Manuscript Info}

\section{Manuscript History}

Received: 03 March 2017

Final Accepted: 04 April 2017

Published: May 2017

\begin{abstract}
Objective: To evaluate oral and manual somatosensory perception in skeletal anterior open bite (SAOB) subjects.

Methods: 14 adults with SAOB were tested for oral and manual stereognostic ability using stimuli of varying shape, texture and density. A custom device was used for two point discrimination (TPD) testing. Skeletal Class I subjects with normal overbite served as controls. Time taken and accuracy of identification were noted for stereognosis tests (ST) and distance for TPD. Mann Whitney U tested for differences in the two groups. Spearman's rank correlation was used for association between variables.

Results: Response time for oral ST ranged from 2seconds to 60seconds in SAOB subjects and 2 seconds to 37 seconds in control group. Response time for manual ST ranged from 2seconds to 40.4 seconds in SAOB subjects and 2seconds to 24seconds in control group. Mann Whitney showed the difference was not statistically significant $(p \leq 0.05)$ between groups in both manual and oral ST. Spearman's correlation test showed no correlation between response time and extent of open bite. Frequency of incorrect responses for ST was greater in SAOB. Minimal errors were noted in identification of texture and maximum for identification of cross shape in ST. TPD ranged from $1 \mathrm{~mm}$ to $5 \mathrm{~mm}$ at different sites.

Conclusions: Impairment in oral sterognostic ability is noted in subjects with SAOB though no association is seen between the severity of SAOB and sterognostic ability. Complexity of test shape was found to have the greatest influence on sensory perception regardless of the presence of SAOB in both manual and oral sensory tests.
\end{abstract}

Copy Right, IJAR, 2017,. All rights reserved.
Corresponding Author:- Prasad Sabarinath. Address:- PhD student, Department of Oral Sciences, University of Otago, Dunedin, NZ. 
The densely innervated oral cavity serves as a source of a rich mélange of sensory input, and the delicate balance between oral sensory input and motor activity forms the basis of normal oral function. Without doubt, abnormal motor activity in the oral region is related to oral dysfunction ${ }^{1}$. Unlike the focus on motor activity and oral function, research on oral sensory awareness is relatively sparse and even less is known about the complex interrelationships between structural abnormalities of the orofacial region and altered oral sensation. Sensory domains that have been investigated in the oral region include stereognostic ability, two-point discrimination (TPD), tactile and temperature perception $^{2}$. Stereognosis denotes the ability of an individual to identify shape, texture and density of objects in the absence of visual or auditory inputs ${ }^{3}$. TPD involves the presentation of pressure stimuli and studying the ability to discriminate them.

Previously researchers have focused on studying oral sensory impairment in subjects with defective speech, blindness, deafness, neurological disorders, edentulous states and orofacial clefts ${ }^{4}$.Misarticulation and lisping during speech, alterations during chewing and differences in deglutition patterns are seen in AOB subjects ${ }^{5-7}$.Structural abnormalities in AOB subjects with a skeletal component include a shorter posterior facial height, increased overall anterior facial height, increased lower facial height in relation to upper anterior face height, increased mandibular plane angles and large gonial angles ${ }^{8,9}$. The altered dentofacial morphology in skeletal anterior open bite (SAOB) subjects also affects the position and movements of the tongue during function ${ }^{10,11}$ The tongue, being one of the most innervated organs in the human body, has a vital role in oral sensory perception. A diminished oral sensory ability has been reported in subjects who have undergone surgical procedures of the tongue ${ }^{12,13}$. Though individuals with anterior open bite (AOB) present with unique aberrations in structure, function and tongue activity, research on oral somatosensory perception in AOB, is minimal ${ }^{14,15}$. Unlike oral sensory ability, manual sensory ability has been studied to a relatively greater extent ${ }^{16-18}$, though rarely in the same cohort ${ }^{19}$.

To gain a deeper insight and address the knowledge gap in this area, additional research is needed. Therefore, the experiments reported in this paper were conducted to compare somatosensory perception in SAOB subjects with skeletal Class I subjects having a normal overbite. Additionally, the study also examined if there was an association between severity of the SAOB and oral somatosensory perception. Lastly, the influence of the shape, texture and density on manual and oral sensory perception were investigated.

\section{Methodology:-}

Test Participants: -

Participants for the study were recruited from subjects who reported seeking orthodontic treatment to the dental clinics during the period from June 2104 to June 2016.Subjects with missing teeth other than third molars, recent surgical procedures of the oral cavity, inflammatory conditions of the orofacial region, craniofacial anomalies, under medication, any history of neurologic or psychological illness were excluded from the study. $14(6 \mathrm{M} \& 8 \mathrm{~F})$ adult subjects, between 18-25 years of age, categorized as having a SAOB based on cephalometric criteria (reduced posterior facial height, increased lower facial height in relation to upper anterior facial height, increased mandibular plane angle and increased gonial angle) formed the study group (Figs 1\&2). Open bite (mm) was measured using a digital caliper on orthodontic study models of the study subjects. An equal number of appropriately age and sex matched skeletal Class I subjects with normal overbite formed the control group. Ethical consent for the research was provided by the institutional research committee and consent to participate was obtained from all subjects.

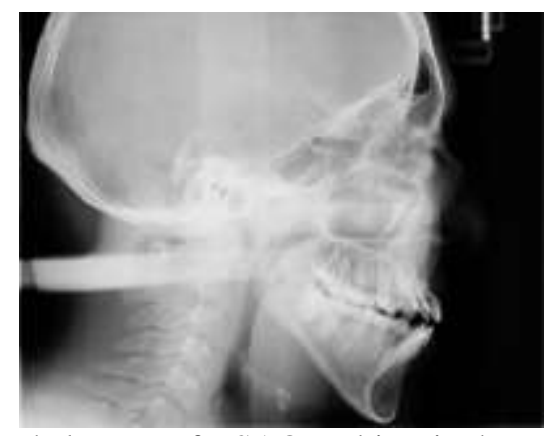

Fig 1:- Cephalogram of a SAOBsubject in the study group. 


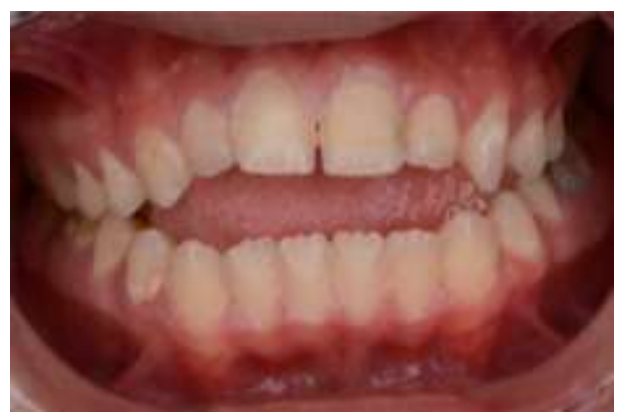

Fig 2:- Intraoral frontal picture of a SAOBsubject in the study group.

\section{Test Apparatus: -}

Six different shapes were used for stereognosis testing (ST)namely square, rectangle, pill, circle, cross and triangle, similar to those used in a previous study ${ }^{20}$.Subsequently, each one of the chosen shapes was fabricated using both non-ferrous metal and acrylic resin corresponding to heavy and light densities respectively. Grooves, at an approximate separation of $0.5 \mathrm{~mm}$ and to a depth of $0.5 \mathrm{~mm}$ were scribed onto the surfaces of the six different metal and six different acrylic shapes resulting in test pieces corresponding to a rough textural quality. The remaining six acrylic and six metal test pieces had their surfaces highly polished corresponding to a smooth textural quality .Hence , a total of 24 test pieces(Table 1)in different combinations of shape, density and texture (Figs 3 \&4) were fabricated for use as test stimuli.12 mil stainless steel orthodontic wires of $5 \mathrm{~cm}$ length were embedded in a block of acrylic at specific degrees of separation (a single wire, $1 \mathrm{~mm}, 2 \mathrm{~mm}, 3 \mathrm{~mm}, 4 \mathrm{~mm}$ and $5 \mathrm{~mm}$ apart).This custom device was used for the TPD tests.

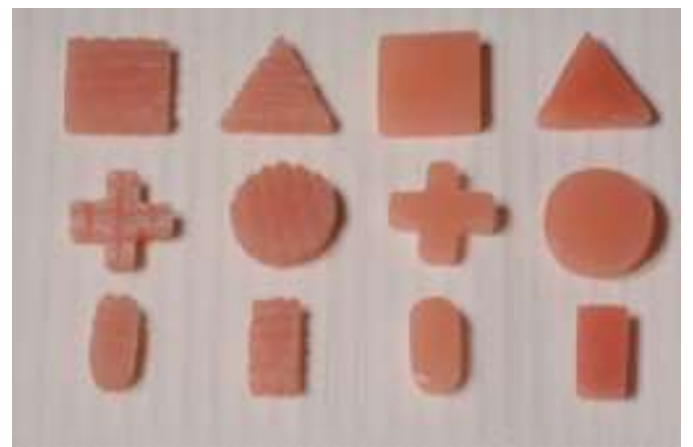

Fig 3:- Acrylic Test Pieces .Rough (two columns on the left) smooth (two columns on the right).

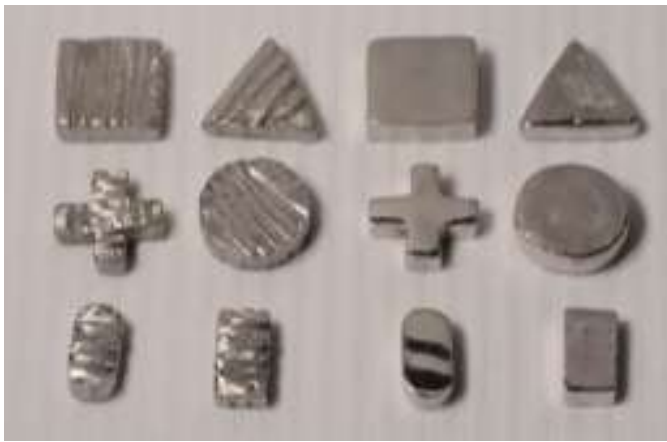

Fig 4:- Mettalic Test Pieces .Rough (two columns on the left) and Smooth (two columns on the right).

\section{Test Procedure: -}

The study design was outlined with clear and precise instructions to all subjects. All testing was performed by the same investigator in a quiet, comfortable room with stable illumination, free of distraction and sources of noise. Tests were performed on subjects two hours after their last meal. During testing, subjects were seated with their eyes closed and each test piece was presented with a tweezer on the tip of the tongue (Fig 5) for oral ST and palm of the dominant hand for manual ST. Each test piece was presented once and a random presentation sequence was 
followed. For oral ST, subjects were instructed to refrain from placing test pieces between their teeth and only permitted to manipulate the test pieces with their tongue and against the palate to aid identification. Subjects were also instructed to be careful not to swallow the test pieces. Once characteristics of the test stimuli were identified, subjects gave a visual cue by raising their hand followed by an oral description of the characteristics perceived, namely, stimulus shape (square, rectangle, pill, circle, cross or triangle), stimulus texture (rough or smooth) and stimulus density (light or heavy). Each response was followed by a fifteen second break prior to the next stimulus presentation. Accuracy of test piece identification and time taken to identify the characteristics of the stimulus were noted for both manual and oral ST. Collectively, 42responses (14 each for shape, density and texture) were noted for each test stimulus in both the manual and oral stereognosis tests. Tests for TPD were done using the custom device on the tip of the tongue, incisive papilla and tip of the middle finger (Figs 6, 7\&8). A random order of stimulus presentation was followed for the TPD tests. The distance at which the orthodontic wires were accurately perceived as two distinct entities was noted for the TPD test. The correctness of their responses was not shared with the study participants at any stage. All test pieces and the TPD device were immersed in disinfectant solution and thoroughly rinsed in water after every use.

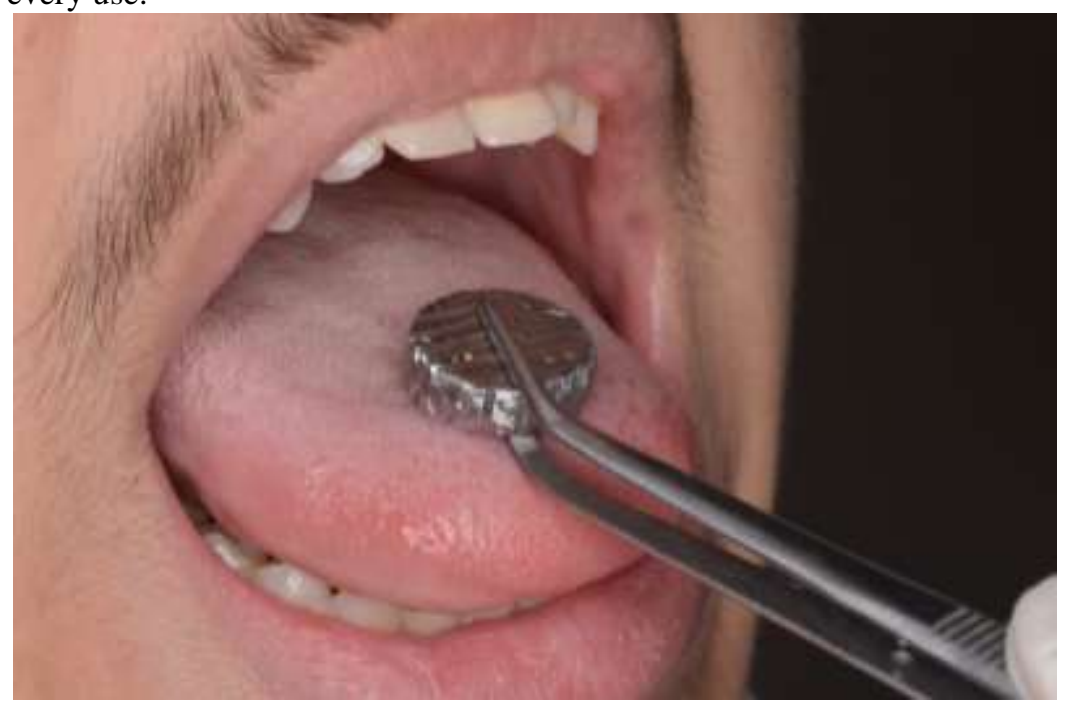

Fig 5:- Oral Sterognosis testing.

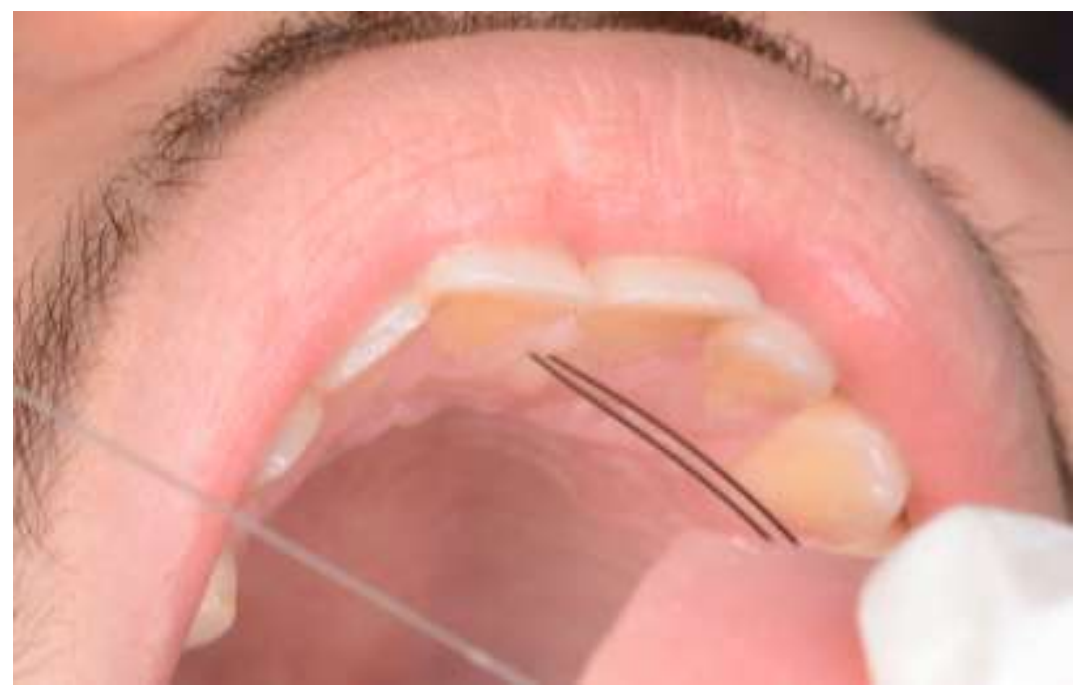

Fig 6: - Intraoral TPD on the incisive papilla. 


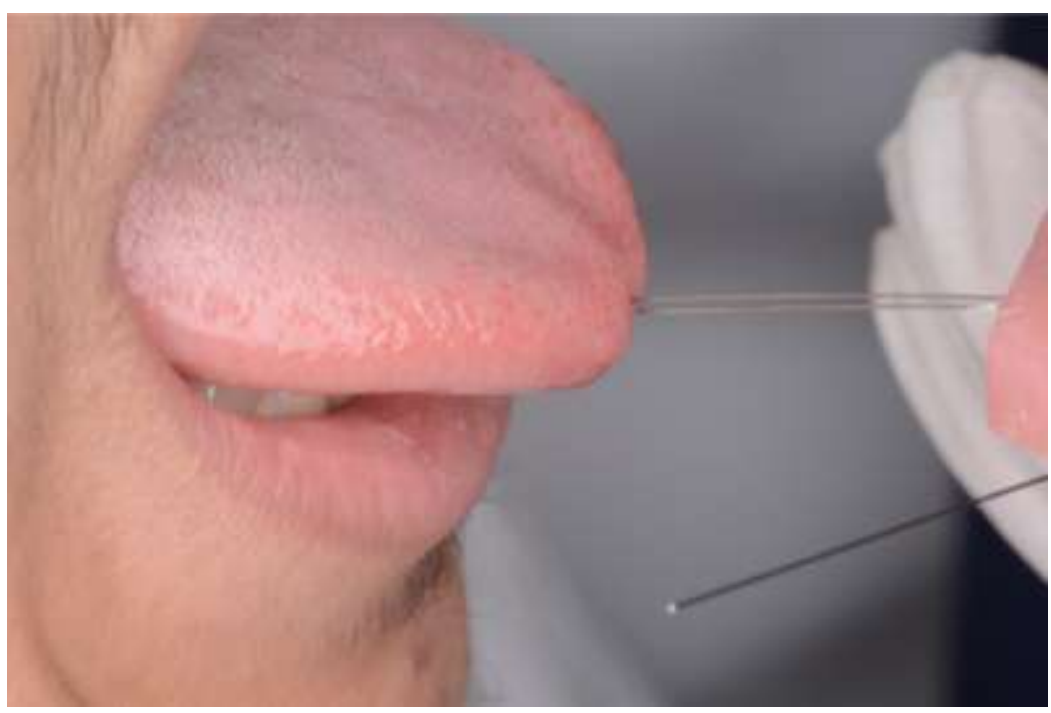

Fig 7: - Intraoral TPD on the tip of the tongue.

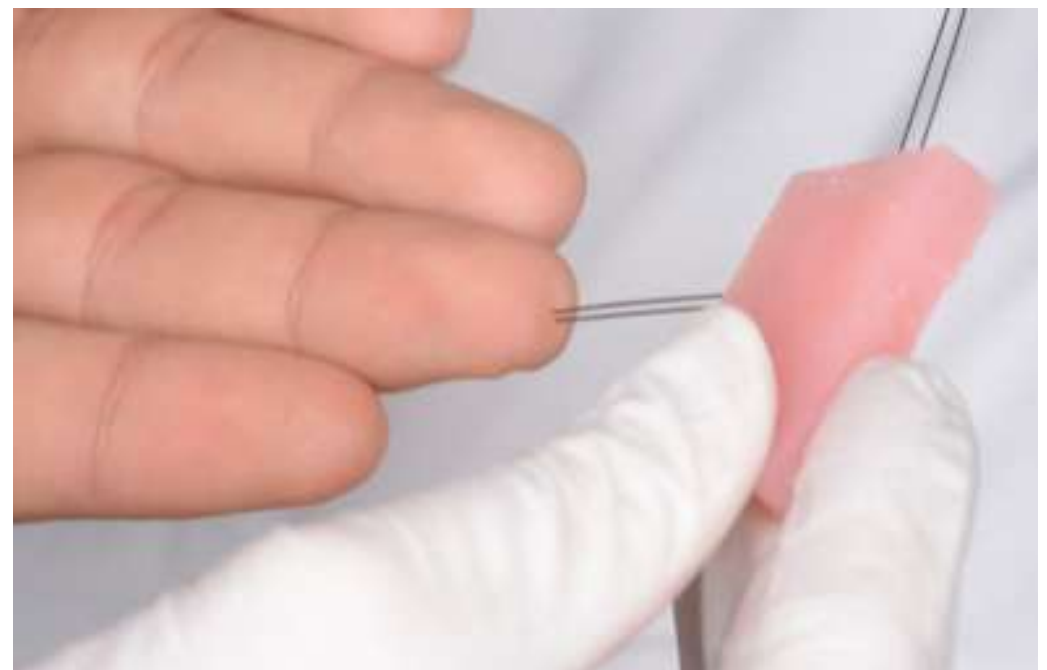

Fig 8: - Manual TPD on the tip of the middle finger

\section{Statistical Analysis: -}

Data was transferred to SPSS Statistics for Windows, Version 22.0 (Chicago,USA:SPSS Inc) for analysis. p - value was set at a significance level of 0.05 . To summarize data, mean \pm standard deviation of time taken for ST and distance for TPD were used. Percentage of incorrect responses for ST was calculated. Mann Whitney U was used to test for differences in the mean time taken for sensory tasks in the different groups after ascertaining the normality of variances by Shapiro-Wilk test. Spearman's rank correlation test assessed the associations between different test variables.

\section{Results:-}

The extent of SAOB in the study group ranged from $3 \mathrm{~mm}$ to $6 \mathrm{~mm}$. Tests of normality showed that data of the oral and manual stereognosis differed from a normal distribution. The time taken to identify characteristics of the presented stimuli in the oral ST ranged from a minimum of 2 seconds to a maximum of 60 seconds for the SAOB subjects and from a minimum of 2 seconds to a maximum of 37 seconds for the skeletal Class I subjects. Though the mean time taken to identify characteristics of the presented stimuli in the oral ST was more in the SAOB subjects in comparison to the skeletal Class I subjects (Table 2), Mann Whitney U test revealed that this difference was not statistically significant $(p>0.05)$. Additionally, Spearman's rank correlation test revealed no correlation between the time taken to identify characteristics of the presented stimuli in the oral ST and the amount of the anterior open bite. 
The time taken to identify characteristics of the presented stimuli in the manual ST ranged from a minimum of 2 seconds to a maximum of 40.4 seconds for the open bite subjects and from a minimum of 2 seconds to a maximum of 24 seconds for the subjects with a normal occlusion. Though the mean time taken to identify characteristics of the presented stimuli in the manual ST was also marginally greater in the SAOB subjects in comparison to the skeletal Class I subjects (Table 3), Mann Whitney U test revealed that this was not statistically significant ( $p>0.05$ ). No correlation was found between the time taken to identify characteristics of the presented stimuli in the oral and the manual ST.

When considering the nature of responses for the different test stimuli, no inaccuracies were noted in the responses for test stimuli Sq M S and Ci M R in both study and control groups during oral ST. Incorrect responses were noted with all other test stimuli. The percentage of incorrect responses was higher in subjects with an SAOB than in subjects with a normal occlusion. The highest number of incorrect responses were for the cross shaped stimulus. Additionally, no inaccurate responses were noted for test stimuli Sq M S, Tr A S, Tr A R, Ci A R, and Ci A S in both study and control groups during manual ST. Incorrect responses were noted with all other test stimuli. The percentage of incorrect responses was again marginally higher in subjects with the SAOB than the normal occlusion subjects. The highest number of incorrect responses during manual ST were also noted in identification for the cross shaped stimulus(Table 4). Additionally, no association was noted in the oral and manual ST for the SAOB and control groups in our study.

Minimal differences were noted for the TPD tests in the both the study and control groups. The minimum distance at which the subjects were able to discern the separation of the orthodontic wires in both the oral and manual TPD tests was $1 \mathrm{~mm}$. The maximum distance for the TPD test were $3 \mathrm{~mm}, 5 \mathrm{~mm}$ and $4 \mathrm{~mm}$ for the tongue tip, incisive papilla and tip of the middle finger respectively (Table 5).No correlation was noted for TPD and ST in both the study and control group.

\section{Discussion:-}

We conducted neurophysiologic experiments to investigate sensory perception of adult subjects with a SAOB. For our research, we employed the TPD and ST for oral somatosensory assessment. Test pieces chosen for stereognosis assessment in the study were similar to those previously used by Kawagishii et $\mathrm{al}^{20}$. They employed a simplified six pieces ST and concluded it to be an effective approach and further recommended this stereognosis assessment in diverse patient populations. During testing, a random order of test stimulus presentation was followed with a fifteen second interval between each presentation to avoid bias arising from respondent fatigue. Subjects with increased vertical craniofacial dimensions with the presence of an $\mathrm{AOB}$, in this study, were seen to have longer response times and greater number of impaired ST responses. AOB is thought to result from interactions of different etiologic factors including non-nutritive habits, airway obstruction and abnormal vertical growth ${ }^{21,22}$. The primary etiological factor in the adult subjects of this study was attributed to unfavorable growth pattern with divergent jaw bases and a resulting absence of contact between the incisors. The etiological factors responsible were primarily growth related for SAOB subjects in this study. Often with these subjects, in the absence of an anterior oral seal due to lack of incisor overlap, a tendency for that space to be occupied by the tongue during swallowing is noted. It is also true that hyperdivergent patients may exhibit a normal or even excessive overbite and patients with normal facial pattern can present with an $\mathrm{AOB}^{23,24}$. Therefore, even if one cannot infer skeletal pattern per se as the sole cause of AOB, the persistent presence of a physical barrier in the form of the tongue preventing incisors from coming into an ideal vertical relationship may possibly be responsible for diminished oral sensory perception in the SAOB subjects. Previous research has also demonstrated a statistically significant inferior sensory ability to perceive shapes and texture in AOB subjects associated with a tongue thrust ${ }^{14}$. In our study, though the response time of the SAOB subjects was longer than the control subjects, the differences were however statistically not significant.

When choosing shapes for ST a fine balance in choosing shapes that enable easy identification but are sufficiently complex to introduce challenges in discrimination is needed. The most errors noted were in identification of the cross shaped stimulus, during both manual and oral ST, irrespective of the presence of an AOB. Of the six shapes used for ST, the cross shape was relatively complex with the presence of multiple sharp corners and angles that may have impaired responses. The relatively smaller surface area of the cross shaped stimulus in comparison to the other shapes may have contributed to diminished oral sensations resulting from the reduced contact with the oral surfaces and thereby introduced errors in perception. In contrast, in the study by Kawagishii et al, no errors were noted in identification of the cross shaped test stimulus by young adults ${ }^{20}$. A possible reason for the differences in this study 
may be because subjects were not only required to assess shape but also of texture and density during a single stimulus presentation.

Combined assessments of test stimuli are a better simulation of real life scenarios rather than split assessments of a specific characteristic during ST. Of the different characteristics tested in our study, the least inaccurate responses were noted for texture perception for both manual and oral ST, irrespective of the AOB. However, this conflicts with the findings of Premkumar et al who noted a high percentage of errors in identification of surface texture in subjects with an anterior open bite ${ }^{14}$. Errors in density identification were intermediate to the errors in shape and texture identification in this study. Size and shape of the various test stimuli were standardized with differences in density based on the material used for fabrication. Even though differences in density of the metal and acrylic test stimuli of the same shape were marginal, subjects were often able to discriminate density. This was in spite of having to make multiple assessments of shape, density and texture during a single stimulus presentation. The study also revealed the possibility of variations existing even among SAOB subject with regard to accuracy of stimulus identification as few did identify test stimuli without any errors. Interestingly, an overall trend for reduction in the time for test stimulus identification was also noted as testing progressed. This may have been the result of subjects becoming more adept in the sensory tasks as testing progressed.

Overall, the response times for manual ST were shorter than oral ST, though response times were again longer in SAOB than normal occlusion subjects. Fewer inaccuracies in responses were also seen in manual ST than oral ST. Fujii et al in their f MRI study found strong activation of somatosensory and motor cortices in both manual and oral $\mathrm{ST}^{11}$. However, differences in activation of the insula and lateral occipital cortex were noted for manual and oral ST. The lack of association seen in the oral and manual sensory tasks of the SAOB and control groups in our study reinforces their conclusions that different neural mechanisms are possibly involved in oral and manual stereognosis. For TPD testing in this study we chose the midline structures of tongue tip, incisive papilla and tip of the middle finger as they exhibit greater sensitivity than more lateral structures. Threshold distance for both manual and oral TPD in all subjects was $5 \mathrm{~mm}$ or lesser. Minor variations with anatomic location may relate to differences in innervation density and differences in receptor field size. When TPD and ST were considered, in general, both subjects with SAOB and normal occlusion had similar thresholds for TPD of the oral and manual areas tested irrespective of the varying stereognostic ability. This is possibly due to the similar basic peripheral neural sensory mechanism of the subjects for the TPD tasks but differences in cortical perception.

However, there are caveats concerning the findings from our research. Firstly, our relatively small sample size is a limitation. However, one also needs to bear in mind that SAOB malocclusion is a relatively rare entity. Secondly, inter -individual variations in response times and accuracy of responses was noted. Lastly, personality traits of individual participants may have played a role in influencing their response times. A "performance anxiety" and greater desire to help and be accurate in their responses may have prompted some participants to work harder resulting in longer response times ${ }^{25}$. Translating results from this research to the clinical setting may help fine tune our strategies to attain better treatment outcomes. In future, it will be interesting to examine the effects of orthodontic treatment on oral somatosensory perception of SAOB subjects.

\section{Conclusion:-}

Impairment in oral sterognostic ability is noted in subjects with SAOB, though no association is seen between the severity of SAOB and sterognostic ability. Complexity of test shape has a major influence on sensory perception regardless of the presence of SAOB in both manual and oral sensory tests . 
Table 1: -Characteristics of the test stimuli

\begin{tabular}{|c|c|c|c|c|}
\hline SHAPE & DENSITY & TEXTURE & WEIGHT (mg) & DIMENSIONS (mm) \\
\hline \multirow[t]{4}{*}{$\mathrm{Sq}$} & \multirow[t]{2}{*}{$\mathrm{A}$} & $S$ & 1.2 & $16 \times 5$ \\
\hline & & $\mathrm{R}$ & 1.1 & $16 \times 5$ \\
\hline & \multirow[t]{2}{*}{ M } & $S$ & 9 & $16 \times 5$ \\
\hline & & $\mathrm{R}$ & 7.7 & $16 \times 5$ \\
\hline \multirow[t]{4}{*}{$\mathbf{R e}$} & \multirow[t]{2}{*}{$\mathrm{A}$} & $\mathrm{S}$ & 0.4 & $15 \times 7.5 \times 5$ \\
\hline & & $\mathrm{R}$ & 0.3 & $15 \times 7.5 \times 5$ \\
\hline & \multirow[t]{2}{*}{ M } & $\mathrm{S}$ & 3.1 & $15 \times 7.5 \times 5$ \\
\hline & & $\mathrm{R}$ & 2.9 & $15 \times 7.5 \times 5$ \\
\hline \multirow[t]{4}{*}{$\operatorname{Tr}$} & \multirow[t]{2}{*}{$\mathrm{A}$} & $\mathrm{S}$ & 0.7 & $15 \times 5$ \\
\hline & & $\mathrm{R}$ & 0.6 & $15 \times 5$ \\
\hline & \multirow[t]{2}{*}{$\mathrm{M}$} & $\mathrm{S}$ & 4.9 & $15 \times 5$ \\
\hline & & $\mathrm{R}$ & 4.8 & $15 \times 5$ \\
\hline \multirow[t]{4}{*}{$\mathbf{C i}$} & \multirow[t]{2}{*}{$\mathrm{A}$} & $\mathrm{S}$ & 1.2 & $16 \times 5$ \\
\hline & & $\mathrm{R}$ & 1 & $16 \times 5$ \\
\hline & \multirow[t]{2}{*}{$\mathrm{M}$} & $\mathrm{S}$ & 8.2 & $16 \times 5$ \\
\hline & & $\mathrm{R}$ & 7.7 & $16 \times 5$ \\
\hline \multirow[t]{4}{*}{$\mathrm{Cr}$} & \multirow[t]{2}{*}{ A } & $\mathrm{S}$ & 0.7 & $16 \times 5$ \\
\hline & & $\mathrm{R}$ & 0.6 & $16 \times 5$ \\
\hline & \multirow[t]{2}{*}{ M } & $\mathrm{S}$ & 3.9 & $16 \times 5$ \\
\hline & & $\mathrm{R}$ & 3.8 & $16 \times 5$ \\
\hline \multirow[t]{4}{*}{$\mathbf{P i}$} & \multirow[t]{2}{*}{ A } & $\mathrm{S}$ & 0.4 & $15 \times 7 \times 5$ \\
\hline & & $\mathrm{R}$ & 0.3 & $15 \times 7 \times 5$ \\
\hline & \multirow[t]{2}{*}{$\mathrm{M}$} & $\mathrm{S}$ & 2.9 & $15 \times 7 \times 5$ \\
\hline & & $\mathrm{R}$ & 2.7 & $15 \times 7 \times 5$ \\
\hline
\end{tabular}

Sq - Square, $\mathbf{R e}$ - Rectangle, Tr -Triangle, Ci - Circle, Cr - Cross, Pi - Pill

A - Acrylic / Light, M -Metal / Heavy

S - Smooth, R- Rough

Table 2: - Time taken for stimulus identification in the oral stereognosis tests

\begin{tabular}{|c|c|c|c|c|c|c|c|}
\hline \multirow{2}{*}{ STIMULUS } & \multicolumn{3}{|c|}{ OPENBITE } & \multicolumn{3}{c|}{ NORMalue } \\
\cline { 2 - 7 } & Min & Max & Mean (SD) & Min & Max & Mean (SD) & 0.606 \\
\hline Sq A S & 4.3 & 19 & $9.1(4.9)$ & 2.5 & 16 & $9.5(4.7)$ & 0.1 \\
\hline Sq A R & 4 & 16.6 & $9.8(4.1)$ & 3.1 & 34.1 & $12.6(9.1)$ & 0.699 \\
\hline Sq M S & 5.1 & 29.1 & $14.9(8.6)$ & 3.5 & 13.5 & $8.5(2.9)$ & 0.065 \\
\hline Sq M R & 2.2 & 50 & $15.1(9.2)$ & 2 & 20.1 & $10.5(5.6)$ & 0.797 \\
\hline Re A S & 3.1 & 23 & $12.7(6.6)$ & 3 & 25.2 & $10.3(6.7)$ & 0.401 \\
\hline Re A R & 4 & 40 & $12.7(11.8)$ & 3.2 & 17 & $8.3(4.1)$ & 0.562 \\
\hline Re M S & 4 & 39 & $13.0(10.9)$ & 3 & 17 & $9.7(4.5)$ & 0.847 \\
\hline Re M R & 3 & 44 & $17.2(13.3)$ & 2 & 17.5 & $9.9(4.6)$ & 0.401 \\
\hline Tr A S & 4 & 45 & $16.1(13.4)$ & 2 & 24 & $11.1(6.3)$ & 0.606 \\
\hline Tr A R & 5 & 28 & $11.4(6.7)$ & 2 & 21 & $10.6(5.6)$ & 0.898 \\
\hline Tr M S & 3 & 60 & $17.5(17.0)$ & 3 & 24 & $10.3(5.9)$ & 0.519 \\
\hline Tr M R & 5.5 & 60 & $18.5(16.6)$ & 2 & 37 & $11.1(9.7)$ & 0.401 \\
\hline Ci A S & 4 & 32 & $9.0(8.4)$ & 4 & 12 & $7.0(2.4)$ & 0.699 \\
\hline Ci A R & 3.4 & 30 & $12.3(8.8)$ & 5.5 & 29 & $11.8(7.0)$ & 1 \\
\hline Ci M S & 3 & 28 & $11.2(6.6)$ & 2 & 15 & $8.2(4.7)$ & 0.562 \\
\hline Ci M R & 5 & 25 & $11(6.7)$ & 3 & 16 & $9.7(4.3)$ & 0.898 \\
\hline Cr A S & 3 & 38 & $15.0(12.0)$ & 3 & 21 & $10.6(5.7)$ & 0.606 \\
\hline Cr A R & 3.7 & 29.1 & $12.0(8.0)$ & 4.1 & 21 & $12.3(6.3)$ & 0.652 \\
\hline Cr M S & 3.1 & 30 & $11.8(8.9)$ & 4 & 11 & $8.0(2.0)$ & 0.606 \\
\hline Cr M R & 3.2 & 21.1 & $11.9(5.7)$ & 2.1 & 18.3 & $9.6(5.4)$ & 0.365 \\
\hline
\end{tabular}




\begin{tabular}{|c|c|c|c|c|c|c|c|}
\hline Pi A S & 3.8 & 30 & $11.0(7.8)$ & 7 & 23.1 & $9.9(4.5)$ & 0.797 \\
\hline Pi A R & 2.6 & 20 & $7.8(5.3)$ & 3 & 36 & $12.9(8.7)$ & 0.076 \\
\hline Pi M S & 3.1 & 60 & $15.4(15.7)$ & 3.4 & 21 & $12.1(5.7)$ & 0.847 \\
\hline Pi M R & 4.5 & 42.1 & $17.0(11.3)$ & 4 & 18.1 & $11.9(4.9)$ & 0.365 \\
\hline
\end{tabular}

Table 3: - Time taken for stimulus identification in the manual stereognosis tests

\begin{tabular}{|c|c|c|c|c|c|c|c|}
\hline \multirow[t]{2}{*}{ STIMULUS } & \multicolumn{3}{|c|}{ OPENBITE } & \multicolumn{3}{|c|}{ NORMAL } & \multirow[t]{2}{*}{ p value } \\
\hline & Min & Max & Mean (SD) & Min & Max & Mean (SD) & \\
\hline $\mathrm{Sq}$ A S & 5 & 23 & $10.3(5.3)$ & 4 & 11 & $6.6(2.2)$ & 0.056 \\
\hline Sq A R & 3 & 31.1 & $10.0(7.8)$ & 2.6 & 8.1 & $5.8(1.6)$ & 0.217 \\
\hline $\mathrm{Sq} \mathrm{M} \mathrm{S}$ & 2 & 11 & $6.4(3.0)$ & 2 & 12.1 & $6.8(2.9)$ & 0.797 \\
\hline $\mathrm{Sq}$ M R & 3.1 & 26 & $10.5(6.6)$ & 4.5 & 10.1 & $7.1(1.7)$ & 0.365 \\
\hline $\operatorname{Re} \mathrm{A} \mathrm{S}$ & 4 & 15 & $8.5(3.6)$ & 2.5 & 13.1 & $6.0(2.9)$ & 0.088 \\
\hline $\operatorname{Re} A R$ & 3.3 & 40.4 & $11.1(10.7)$ & 4.6 & 12.2 & $6.3(2.1)$ & 0.243 \\
\hline $\operatorname{Re} M S$ & 4.1 & 15 & $8.4(3.4)$ & 4 & 10.1 & $6.2(1.9)$ & 0.088 \\
\hline Re M R & 3.3 & 21 & $9.7(5.5)$ & 3.4 & 11 & $6.6(2.1)$ & 0.217 \\
\hline $\operatorname{Tr} \mathrm{A} \mathrm{S}$ & 3.1 & 10 & $6.5(2.4)$ & 2.1 & 8.5 & $4.9(2.1)$ & 0.076 \\
\hline Tr A R & 3 & 18 & $8.3(4.7)$ & 3.1 & 9 & $6.0(2.0)$ & 0.27 \\
\hline $\operatorname{Tr} M S$ & 4 & 21 & $9.0(5.3)$ & 4 & 12 & $6.4(2.2)$ & 0.332 \\
\hline TrM R & 3 & 29 & $11.1(8.2)$ & 2.7 & 11 & $7.2(2.6)$ & 0.478 \\
\hline $\mathrm{Ci}$ A S & 3 & 14 & $6.9(3.6)$ & 3.1 & 21 & $6.7(5.1)$ & 0.652 \\
\hline Ci A R & 2.1 & 17 & $8.0(4.4)$ & 2.6 & 9.1 & $6.4(2.1)$ & 0.699 \\
\hline Ci M S & 3 & 15 & $6.5(3.4)$ & 3.1 & 10.1 & $5.6(2.1)$ & 0.748 \\
\hline Ci M R & 2.1 & 16 & $7.3(4.6)$ & 3.2 & 15 & $6.7(3.3)$ & 0.949 \\
\hline CrA S & 4 & 21 & $9.0(5.6)$ & 5 & 21 & $10.1(6.0)$ & 0.652 \\
\hline $\mathrm{Cr} A \mathrm{R}$ & 3 & 17 & $8.9(4.2)$ & 2.3 & 24 & $10.1(6.7)$ & 0.898 \\
\hline Cr M S & 4 & 18.6 & $8.4(5.1)$ & 4 & 16.1 & $8.6(3.4)$ & 0.438 \\
\hline Cr M R & 3.1 & 21 & $10.9(6.9)$ & 2.3 & 17 & $10.6(4.9)$ & 1 \\
\hline Pi A S & 2.1 & 13.2 & $7.1(3.2)$ & 3.5 & 10 & $7.2(2.4)$ & 0.949 \\
\hline Pi A R & 2.1 & 11.2 & $7.1(2.3)$ & 4.1 & 9 & $7.1(1.8)$ & 0.949 \\
\hline Pi M S & 3.1 & 22 & $9.9(5.5)$ & 5 & 11.1 & $7.7(1.6)$ & 0.365 \\
\hline Pi M R & 4 & 18 & $9.9(4.8)$ & 4 & 14.2 & $8.3(3.2)$ & 0.478 \\
\hline
\end{tabular}

Table 4: - Percentage of errors in identification of shape(S), density(D) and texture(T) in ST

\begin{tabular}{|c|c|c|c|c|c|c|c|c|c|c|c|c|}
\hline \multirow[t]{3}{*}{ STIMULUS } & \multicolumn{6}{|c|}{ ORAL } & \multicolumn{6}{|c|}{ MANUAL } \\
\hline & \multicolumn{3}{|c|}{ OPEN BITE } & \multicolumn{3}{|c|}{ NORMAL } & \multicolumn{3}{|c|}{ OPEN BITE } & \multicolumn{3}{|c|}{ NORMAL } \\
\hline & $\mathbf{S}$ & D & $\mathbf{T}$ & $\mathbf{S}$ & D & $\mathbf{T}$ & $\mathbf{S}$ & D & $\mathbf{T}$ & $\mathbf{S}$ & D & $\mathbf{T}$ \\
\hline $\mathrm{Sq}$ A S & 14.3 & 21.4 & 0.0 & 0.0 & 7.1 & 7.1 & 0.0 & 0.0 & 0.0 & 7.1 & 7.1 & 0.0 \\
\hline $\mathrm{Sq}$ A R & 7.1 & 14.3 & 7.1 & 7.1 & 7.1 & 7.1 & 7.1 & 0.0 & 0.0 & 7.1 & 7.1 & 0.0 \\
\hline Sq M S & 0.0 & 0.0 & 0.0 & 0.0 & 0.0 & 0.0 & 0.0 & 0.0 & 0.0 & 0.0 & 0.0 & 0.0 \\
\hline $\mathrm{Sq} \mathrm{M} \mathrm{R}$ & 14.3 & 0.0 & 7.1 & 7.1 & 0.0 & 14.3 & 0.0 & 7.1 & 0.0 & 0.0 & 0.0 & 0.0 \\
\hline $\operatorname{Re} \mathrm{A} \mathrm{S}$ & 14.3 & 14.3 & 0.0 & 14.3 & 0.0 & 0.0 & 7.1 & 7.1 & 0.0 & 0.0 & 0.0 & 0.0 \\
\hline $\operatorname{Re} A \mathrm{R}$ & 28.6 & 0.0 & 0.0 & 0.0 & 0.0 & 0.0 & 0.0 & 0.0 & 0.0 & 0.0 & 0.0 & 0.0 \\
\hline Re M S & 28.6 & 21.4 & 0.0 & 0.0 & 7.1 & 0.0 & 0.0 & 28.6 & 0.0 & 0.0 & 14.3 & 0.0 \\
\hline Re M R & 14.3 & 28.6 & 0.0 & 0.0 & 0.0 & 0.0 & 7.1 & 21.4 & 0.0 & 0.0 & 7.1 & 0.0 \\
\hline $\operatorname{Tr} \mathrm{A} \mathrm{S}$ & 7.1 & 14.3 & 0.0 & 0.0 & 7.1 & 0.0 & 0.0 & 7.1 & 0.0 & 0.0 & 0.0 & 0.0 \\
\hline $\operatorname{Tr} A \mathrm{R}$ & 7.1 & 7.1 & 0.0 & 7.1 & 14.3 & 0.0 & 0.0 & 0.0 & 0.0 & 0.0 & 0.0 & 0.0 \\
\hline $\operatorname{Tr} \mathrm{M} \mathrm{S}$ & 14.3 & 21.4 & 7.1 & 14.3 & 7.1 & 0.0 & 7.1 & 21.4 & 7.1 & 0.0 & 7.1 & 0.0 \\
\hline $\operatorname{Tr} M \mathrm{R}$ & 7.1 & 7.1 & 7.1 & 7.1 & 0.0 & 0.0 & 0.0 & 7.1 & 0.0 & 0.0 & 14.3 & 7.1 \\
\hline Ci A S & 0.0 & 14.3 & 0.0 & 0.0 & 14.3 & 0.0 & 0.0 & 7.1 & 0.0 & 0.0 & 7.1 & 0.0 \\
\hline $\mathrm{Ci} \mathrm{A} \mathrm{R}$ & 7.1 & 14.3 & 7.1 & 0.0 & 28.6 & 0.0 & 0.0 & 14.3 & 7.1 & 0.0 & 0.0 & 0.0 \\
\hline $\mathrm{Ci} \mathrm{M} \mathrm{S}$ & 0.0 & 14.3 & 0.0 & 0.0 & 14.3 & 0.0 & 0.0 & 0.0 & 7.1 & 0.0 & 0.0 & 0.0 \\
\hline Ci M R & 0.0 & 0.0 & 0.0 & 0.0 & 0.0 & 0.0 & 0.0 & 14.3 & 0.0 & 0.0 & 0.0 & 0.0 \\
\hline Cr A S & 42.9 & 0.0 & 7.1 & 21.4 & 7.1 & 0.0 & 35.7 & 0.0 & 0.0 & 21.4 & 0.0 & 0.0 \\
\hline
\end{tabular}




\begin{tabular}{|c|c|c|c|c|c|c|c|c|c|c|c|c|}
\hline Cr A R & 35.7 & 7.1 & 0.0 & 35.7 & 14.3 & 0.0 & 28.6 & 7.1 & 0.0 & 28.6 & 0.0 & 0.0 \\
\hline Cr M S & 35.7 & 35.7 & 0.0 & 42.9 & 7.1 & 7.1 & 28.6 & 14.3 & 0.0 & 21.4 & 7.1 & 0.0 \\
\hline Cr M R & 42.9 & 35.7 & 7.1 & 42.9 & 14.3 & 0.0 & 35.7 & 21.4 & 0.0 & 28.6 & 7.1 & 0.0 \\
\hline Pi A S & 21.4 & 0.0 & 0.0 & 7.1 & 28.6 & 0.0 & 21.4 & 0.0 & 0.0 & 0.0 & 0.0 & 7.1 \\
\hline Pi A R & 21.4 & 0.0 & 0.0 & 14.3 & 7.1 & 0.0 & 14.3 & 0.0 & 0.0 & 0.0 & 0.0 & 7.1 \\
\hline Pi M S & 21.4 & 35.7 & 0.0 & 28.6 & 7.1 & 0.0 & 21.4 & 28.6 & 0.0 & 0.0 & 7.1 & 0.0 \\
\hline Pi M R & 28.6 & 35.7 & 0.0 & 14.3 & 7.1 & 0.0 & 28.6 & 35.7 & 0.0 & 0.0 & 7.1 & 0.0 \\
\hline
\end{tabular}

Table 5: - Distance at which Two Point Discrimination was noted (mm)

\begin{tabular}{|c|c|c|c|c|c|c|}
\hline \multirow{2}{*}{ AREA } & \multicolumn{3}{|c|}{ OPENBITE } & \multicolumn{3}{c|}{ NORMAL } \\
\cline { 2 - 7 } & Min & Max & Mean (SD) & Min & Max & Mean(SD) \\
\hline Tongue tip & 1 & 3 & $2.3(0.6)$ & 2 & 3 & $2.3(0.4)$ \\
\hline Incisive papilla & 1 & 4 & $2.1(0.9)$ & 1 & 5 & $2.8(1.0)$ \\
\hline Tip of middle finger & 1 & 4 & $2.8(0.8)$ & 1 & 3 & $2.3(0.8)$ \\
\hline
\end{tabular}

\section{References:-}

1. Graber TM. The "three M's": Muscles, malformation, and malocclusion. Am J Orthod.1963;49 (6):418-450

2. Boliek CA, Rieger JM, Li SY, Mohamed Z, Kickham J, Amundsen K. Establishing a reliable protocol to measure tongue sensation. J Oral Rehabil. 2007; 34(6):433-41.

3. Botez MI, Botez T, Olivier M. Handbook of clinical neurology. Volume 1 Clinical Neurophysiology. Philadelphia: Elsevier Science Publisher; 1985. p. 45.

4. Jacobs R, Serhal CB, van Steenberghe D. Oral stereognosis: a review of the literature. Clin Oral Investig. 1998; 2(1):3-10.

5. Jeryl D. English, P.H. Buschang, G.S. Throckmorton, Does Malocclusion Affect Masticatory Performance? Angle Orthod .2002;72:21-27

6. Laine T. Malocclusion traits and articulatory components of speech. Eur J Orthod 1992; 14:302-9.

7. Piancino M, Isola G, Merlo A, Dalessandri D, Debernardi C, Bracco P. Chewing pattern and muscular activation in open bite patients. J Electromyogr Kinesiol. 2012;22(2):273-9

8. Cangialosi TJ. Skeletal morphologic features of anterior open bite. Am J Orthod. 1984; 85(1):28-36.

9. Subtelny HD, Sakuda M. Open bite: diagnosis and treatment. Am J Orthod. 1964 ;50(5):337-58.

10. Lowe AA, Johnson WD. Tongue and jaw muscle activity in response to mandibular rotations in a sample of normal and anterior open-bite subjects. Am J Orthod. 1979; 76:565-576.

11. Fujii, R., Takahashi, T., Toyomura, A., Miyamoto, T., Ueno, T., Yokoyama, A. Comparison of cerebral activation involved in oral and manual stereognosis. J. Clin Neurosci. 2011; 18 (11), 1520-23.

12. Elfring TT, Boliek CA, Seikaly H, Harris J, Rieger JM. Sensory outcomes of the anterior tongue after lingual nerve repair in oropharyngeal cancer. J Oral Rehabil. 2012; 39(3):170-81.

13. Ingervall B, Schmoker R. Effect of surgical reduction of the tongue on oral stereognosis, oral motor ability, and the rest position of the tongue and mandible. Am J Orthod Dentofacial Orthop 1990; 97:58-65

14. Premkumar S, Venkatesan A S, Rangachari S. Altered oral sensory perception in tongue thrusters with an anterior open bite. Eur J Orthod. 2011; 33(2):139-42.

15. Dahan JS, Lelong O, Celant S, Leysen V. Oral perception in tongue thrust\& other oral habits. Am J Orthod Dentofacial Orthop. 2000; 118(4):385-91.

16. Miquee A, Xerri C, Rainville C, et al. Neuronal substrates of haptic shape encoding and matching: a functional magnetic resonance imaging study. Neuroscience .2008; 152:29-39.

17. Bodegard A, Geyer S, Grefkes C, et al. Hierarchical processing of tactile shape in the human brain. Neuron.2001; 31:317-28.

18. Bonda E, Petrides M, Evans A. Neural systems for tactual memories. J Neurophysiol.1996; 75:1730-7.

19. Fujii, R., Takahashi, T., Toyomura, A., Miyamoto, T., Ueno, T., Yokoyama, A. Comparison of cerebral activation involved in oral and manual stereognosis. J. Clin Neurosci. 2011; 18 (11), 1520-23.

20. Kawagishi S, Tanaka T, Shimodozono M, Yoshino K. Simplifying the assessment of stereognostic ability of the tongue in elderly subjects using six selected test pieces. Aging Sci. 2013; 1: 111.

21. Harvold EP, Tomer BS, Vagervik K, Chierici G. Primate experiments on oral respiration. Am J Orthod. 1981; 79(4):359-72.

22. Subtelny HD, Sakuda M. Open bite: diagnosis and treatment. Am J Orthod. 1964; 50(5):337-58. 
23. Dung J, Smith R. Cephalometric and clinical diagnosis of open bite tendency. Am J Orthod. 1998; 94(6):48490.

24. Shapiro PA. Stability of open bite treatment. Am J Orthod Dentofacial Orthop. 2002; 121(6):566-8.

25. Mandrick K, Peysakhovich V, Rémy F, Lepron E, Causse M. Neural and psychophysiological correlates of human performance under stress and high mental workload. Biol Psychol. 2016;121(Pt A):62-73. 